\title{
Into the Secrets of Jazz Arranging: \\ Chromatic Scale in Different Harmonic Contexts
}

\author{
Carmine Cataldo
}

How to cite this paper: Cataldo, C.; Into the Secrets of Jazz Arranging: Chromatic Scale in Different Harmonic Contexts. Journal of Art and Design 2021, 1(1), 1-10. https://doi.org/10.31586/jad.2021.010 101

Received: May 03, 2021 Accepted: May 11, 2021 Published: May 12, 2021

Copyright: (c) 2021 by the authors. Submitted for possible open access publication under the terms and conditions of the Creative Commons Attribution (CC BY) license (http://creativecommons.org/licenses /by/4.0/).
PhD in Mechanical Engineering, MD in Disciplines of Jazz and Improvisation - Jazz Piano

*Correspondence: catcataldo@hotmail.it

\begin{abstract}
Herein we introduce a reliable and effective method, allowing any musician, regardless of the theoretical background, to carry out a 4-way jazz harmonization of whatever melodic progression almost instantly, with few exceptions. Many jazz students experience a deep frustration in dealing with the harmonization of non-diatonic notes. Sometimes, moreover, a coherent harmonization of the aforementioned notes can turn out to be a very challenging task even for extremely skilled professionals. In this paper, the harmonization of the chromatic scale in different harmonic contexts is accurately discussed, by resorting to the well-known concepts of harmonic functions, tonicization, chromatic and diatonic parallelism, and auxiliary chords. All the chords are labelled so as to allow the reader to immediately understand their role in the particular harmonic context. Consequently, the procedure essentially translates into an optimization of the "harmonic flow".
\end{abstract}

Keywords: Jazz Arranging, Jazz Composing, Chromatic Scale, Harmonization, 4-Way Close, Tonicization, Auxiliary Chords, Non-Diatonic Notes

\section{Introduction}

We propose different harmonizations of the chromatic scale in different contexts. In detail, we accurately discuss major seventh, dominant seventh and minor seventh chords, briefly mentioning the half-diminished chords.

By starting from the explicit writing of the so-called 4-Way Close voicings [1], built on the conventional jazz scales (such as the Be-Bop Major and Minor Scales) [2], several useful harmonizations of the chromatic scale are herein explicitly deduced and thoroughly analyzed.

In jazz, the fundamental "rules" characterizing an effective harmonization process are the following:

a. The motion of the parts must not be contrary: consequently, it can be parallel, direct or oblique;

b. It is advisable (not compulsory) to avoid clusters involving external parts;

c. Consistent with the concept of 4-Way Close (from now onwards 4WC), the interval between the external voices must be no greater than a major seventh;

d. Any major seventh, minor seventh and dominant seventh chord can be tonicized by resorting to the corresponding (functioning) secondary dominant (e.g., G7 can be preceded by D7) $[1,3,4]$

e. Any dominant seventh chord, if it is regarded as provided with an added flat ninth, can be replaced by a diminished chord built on the (major) 3rd, the 5th, the (minor) 7th, and the flat 9 th [5];

f. Any major seventh and dominant seventh chord can be preceded by the corresponding diminished auxiliary chord (e.g., G7 can be preceded by Gdim7) [6]; 
g. It is possible to harmonize different notes, even if they are adjacent, by resorting to the same kind of chord. The procedure is carried out by exploiting the inversions of the chord and/or adding tensions, altered or not;

$h$. In order to guarantee coherence to the harmonization, the functions of the chords [7] should be always taken into account.

For the sake of clarity, we underline how the harmonized notes coincide with the upper voices.

\section{Major Seventh Chords}

\subsection{Harmonic Flows}

The suggested harmonic flows are shown in the following diagram (Figure 1):

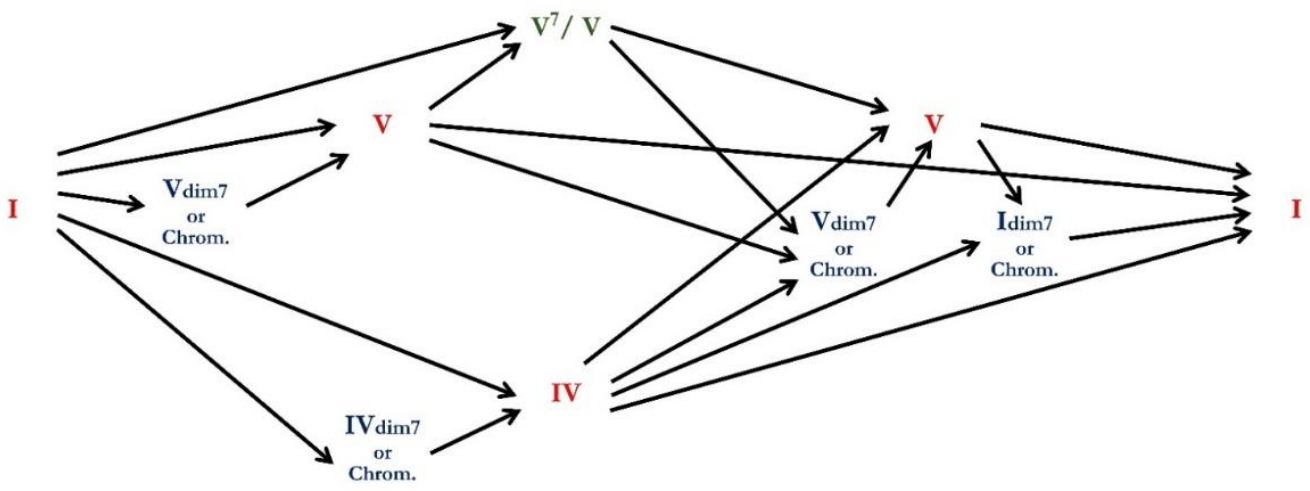

Fig.1 - Major Seventh Chords: Suggested Harmonic Flows

\subsection{Voicings on Conventional Scales: Ionian and Be-Bop Major}

Staves (1) and (2) contain two harmonizations of the C Ionian Scale [7], carried out by resorting to the Diatonic Parallelism $[3,8,9]$. The first harmonization, devoid of clusters involving external voices and since harmonically coherent, can be used as it is. The second harmonization, nothing but a series of tertian quadrichords, cannot be exploited in its entirety, but generates some very useful voicings.

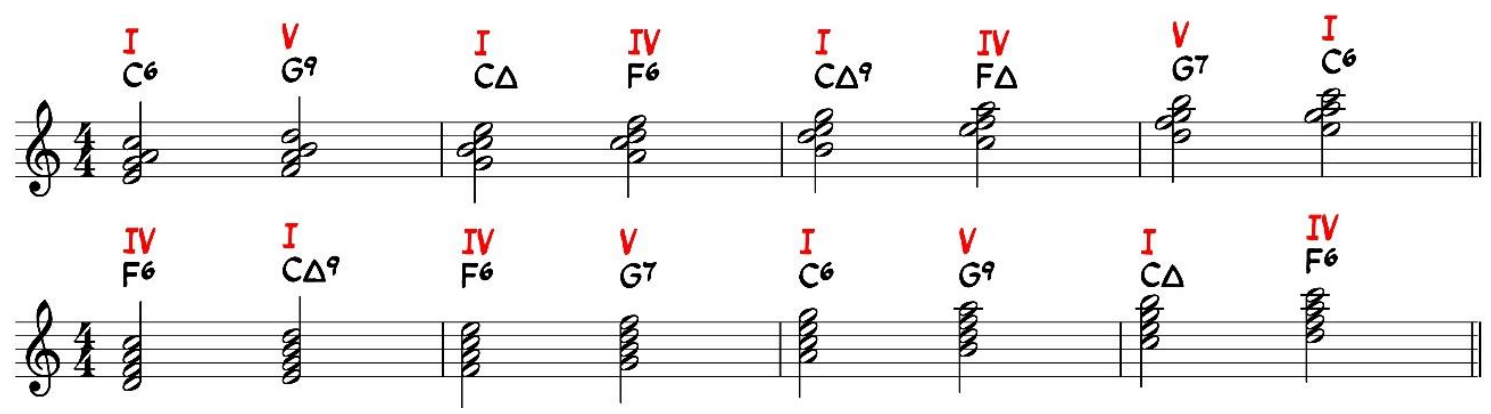

Staff (3) contains the conventional 4WC built on the C Be-Bop Major Scale [2,10]:

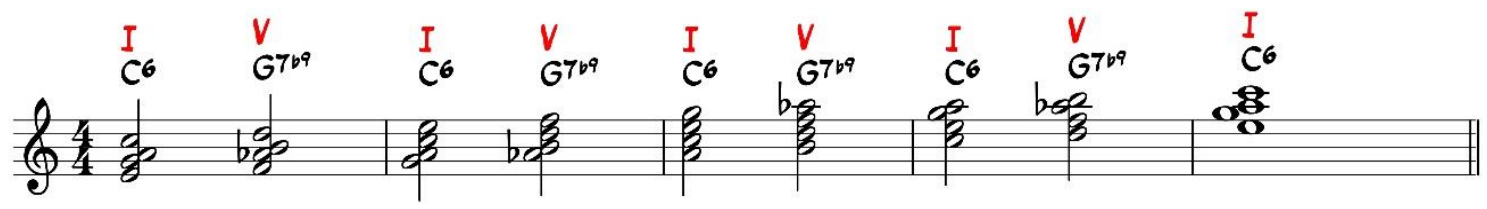


From the previous, by resorting to some of the voicings in Staves (1) and (2), we obtain the following modified 4WC for the C Be-Bop Major Scale [3], devoid of clusters involving external voices:

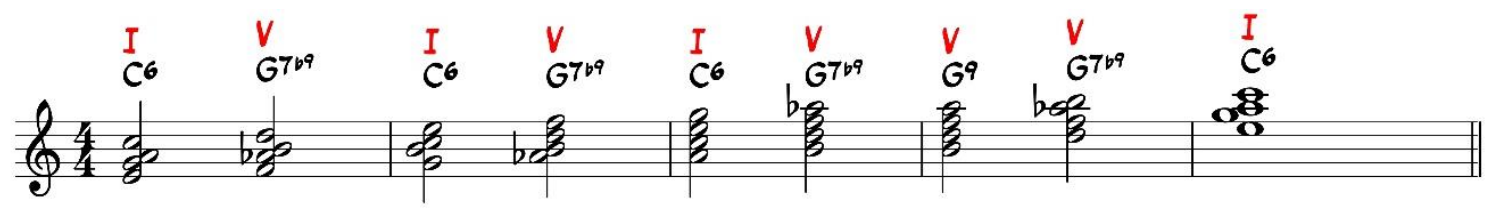

Finally, by modifying the voicing built on the dominant (the 5th), from the previous we obtain the following alternative harmonization:

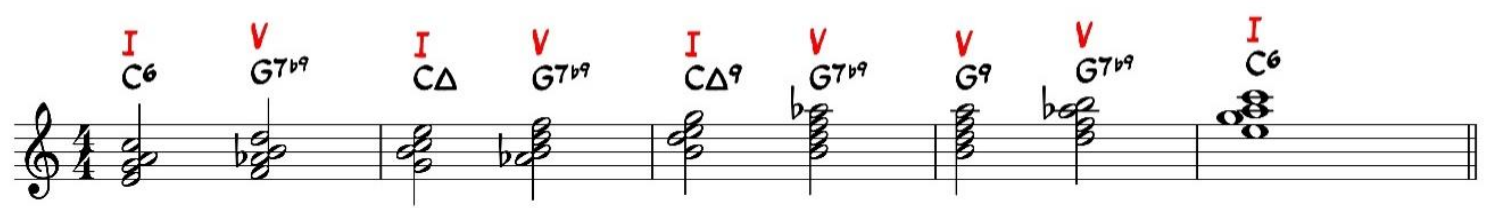

The voicing built on the dominant, labelled as $C \Delta^{9}$, has been taken from Staff (1).

\subsection{Chromatic Scales}

From Staff (1), by resorting to the Chromatic Parallelism [3,9], we obtain:

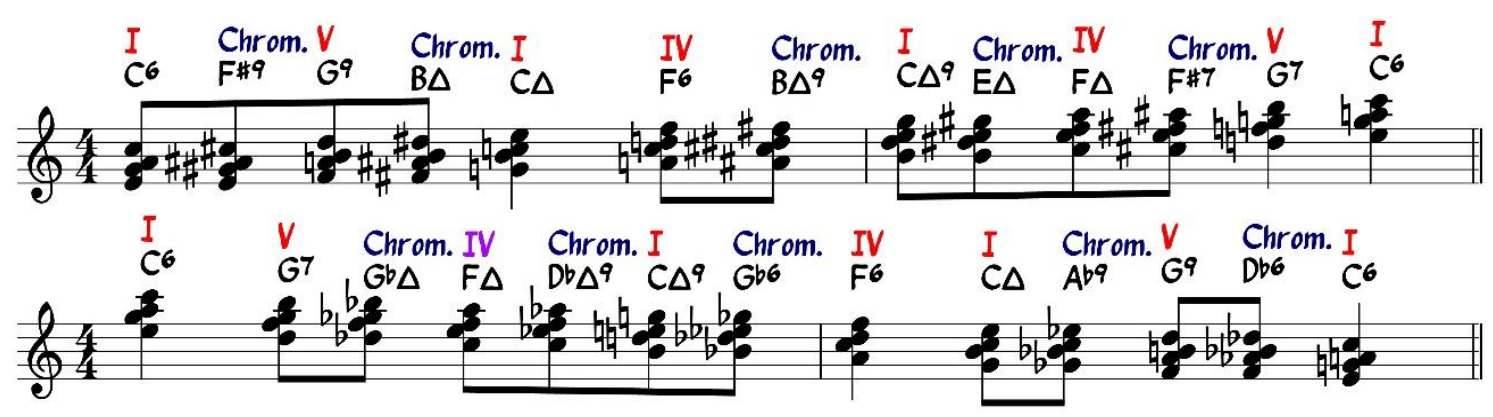

The descending motion in Staff (7), although acceptable, is not harmonically coherent. As an alternative, we can substitute the voicing built on the submediant (the 6th) and, as a consequence, the one built on the minor 6th, thereby obtaining the following solution:

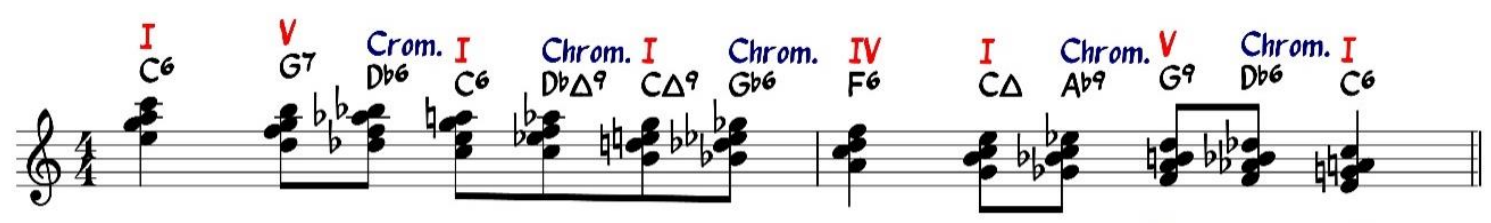

The voicing built on the dominant, labelled as C6, has been taken from Staff (3). Unfortunately, it contains a cluster involving the upper voice. Consequently, the choice between the two options exclusively depends on the priorities of the arranger.

Now, from Staff (4), by resorting to Diminished Auxiliary Chords [6] and Tonicization $[1,3,4,9]$, we easily deduce the underlying alternative harmonization: 


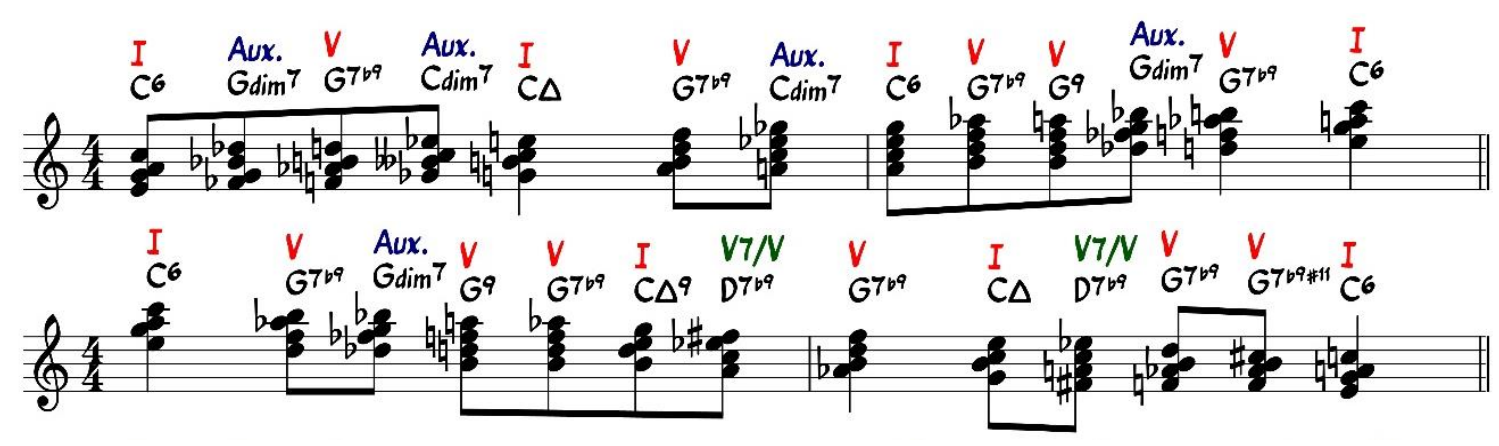

\subsection{Quasi-Static Harmonization}

With the expression "Quasi-Static" harmonization we refer to a series of voicings built by maximizing the common notes between consecutive chords. This kind of procedure turns out to be extremely useful to pianists interested in the so-called "Locked-Hands" technique [1].

We propose the following solution, built by starting from Staff (5):

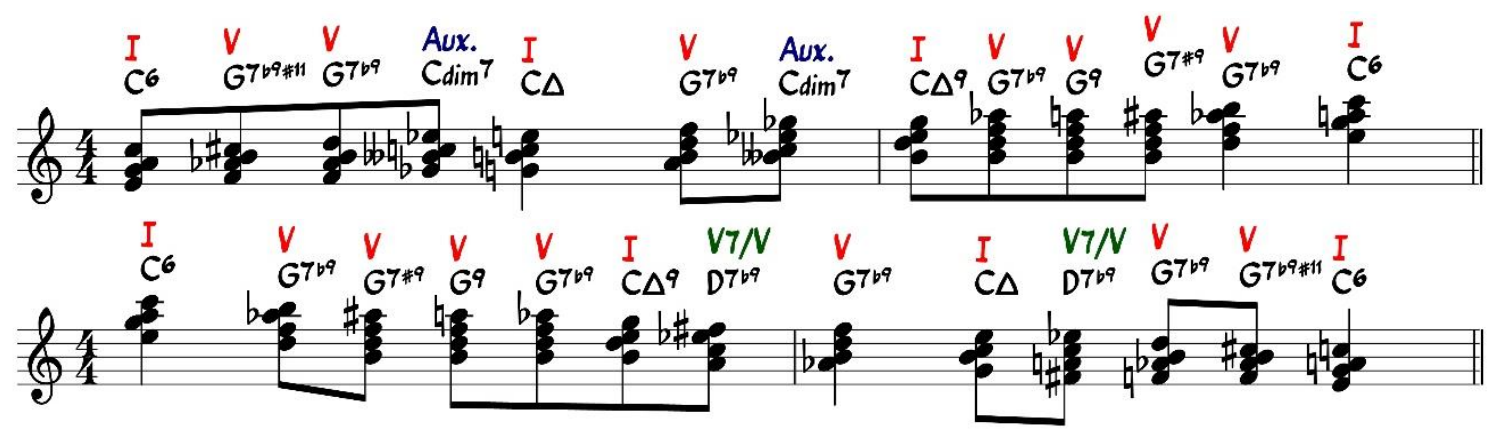

\subsection{Practical Applications}

We propose three solutions for the harmonization of the so-called Cataldo's Fundamental "Sentence" for Major Seventh Chords [10,11]:

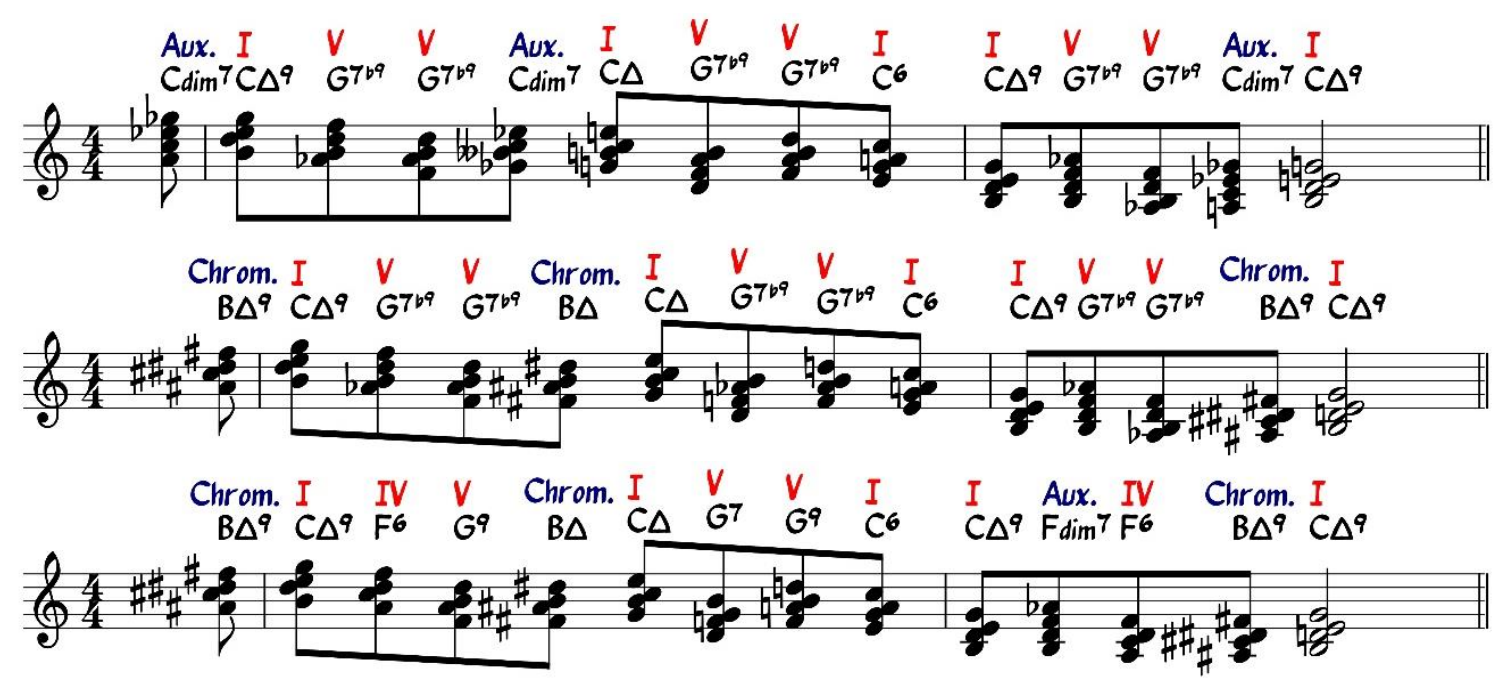

\section{Dominant Seventh Chords}

\subsection{Harmonic Flows}

The suggested harmonic flows are shown in the following diagram (Figure 2): 


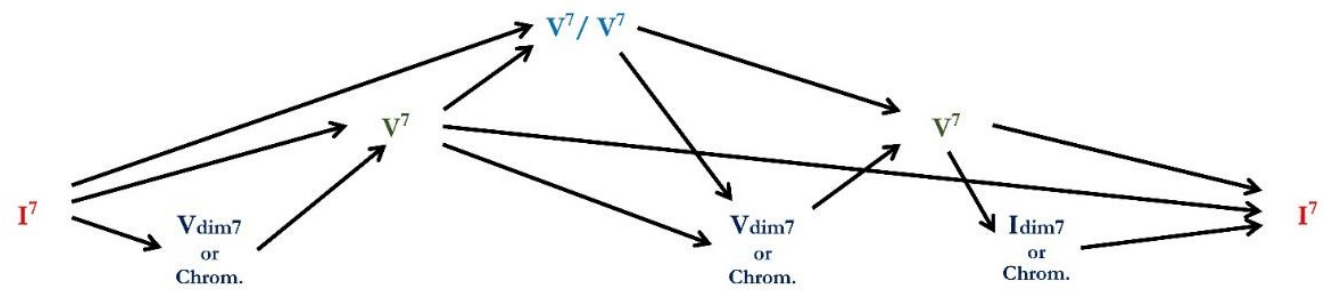

Fig.2 - Dominant Seventh Chords: Suggested Harmonic Flows

3.2. Voicings on Conventional Scales: Be-Bop Dominant and Be-Bop Mixolydian b6 In Staff (16) we have the conventional 4WC of the C Be-Bop Dominant Scale [2]:

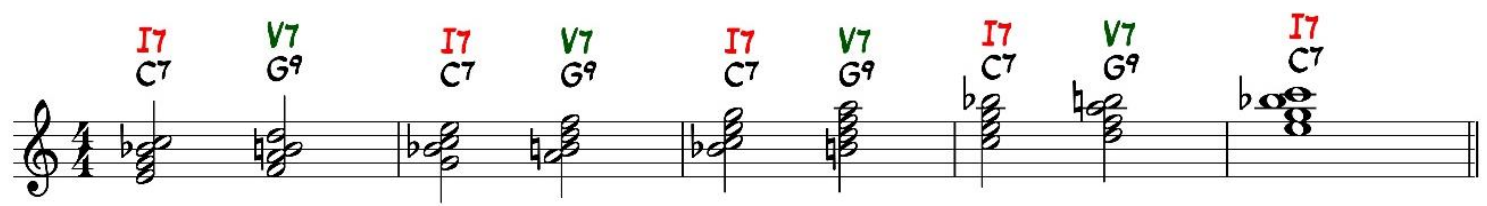

From the previous, by removing the clusters involving the external parts of the voicings built on the mediant (3rd), the dominant (5th) and the sub-tonic (minor 7th), the following form is easily obtained:

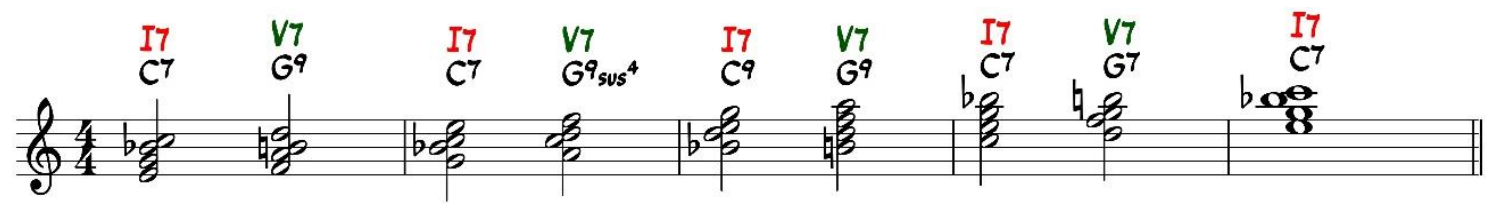

Staff (18) contains the well-known 4WC for the C Be-Bop Mixolydian b6 [3]:

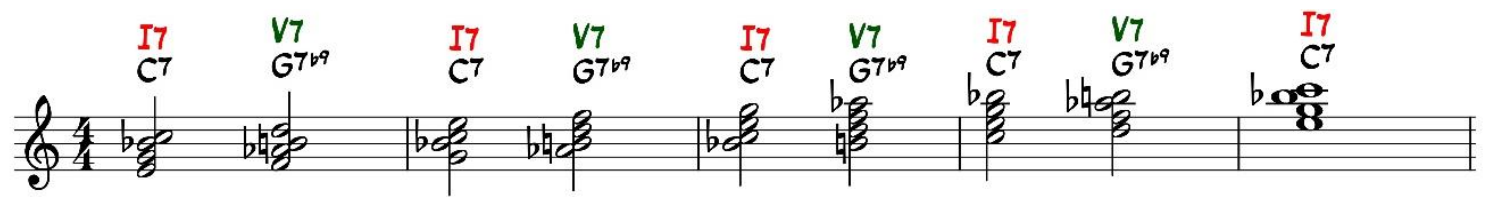

From the previous, by removing the cluster involving the lower part of the voicing built on the dominant (5th), the following alternative form is easily obtained:

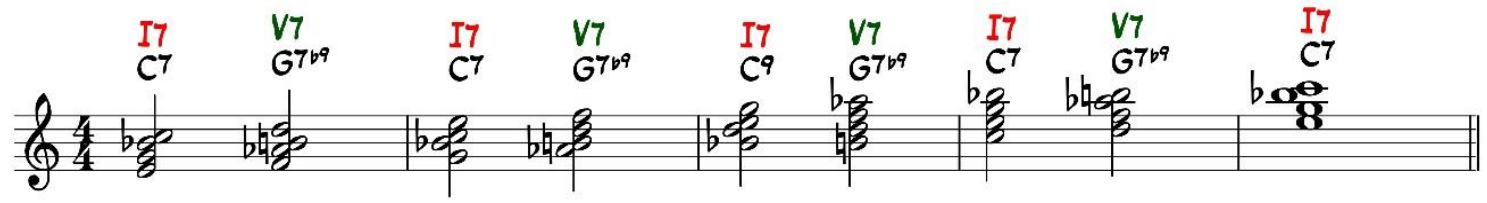

\subsection{Chromatic Scale}

From Staff (17), by resorting to Chromatic Parallelism [3,9] and Tonicization (all the chords labelled as V7 arise from a Secondary Dominant Substitution) [12,13], we have:

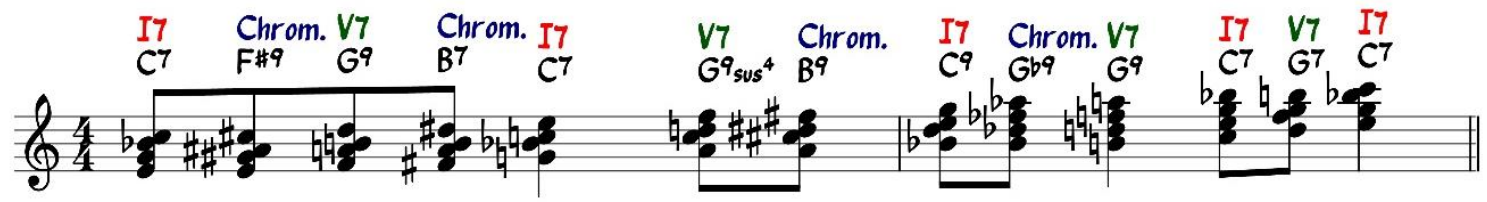




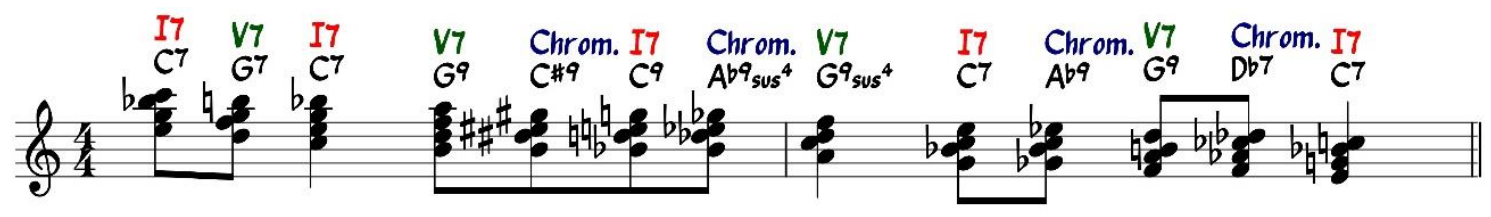

From Staff (19), by resorting to Chromatic Parallelism [3,9], Tonicization and Diminished Auxiliary Chords [6], we obtain the following alternative solution:

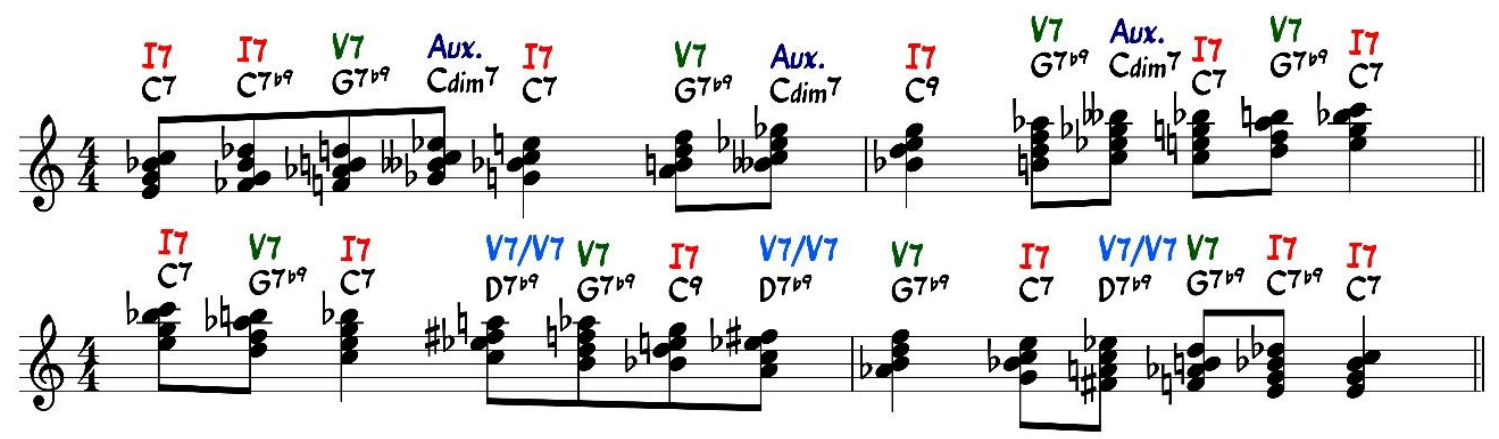

\subsection{Quasi-Static Harmonization}

We propose the following solution, built by slightly modifying Staves (22) and (23):

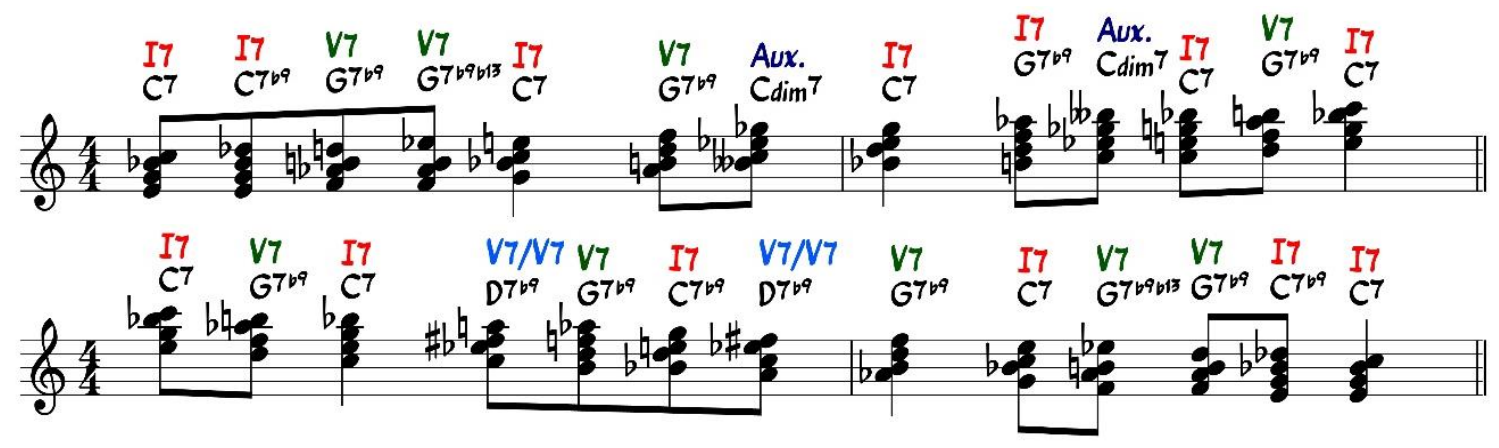

The following represents an extreme version of the previous, predominantly addressed to pianists interested in the "Locked-Hands" technique [1]:

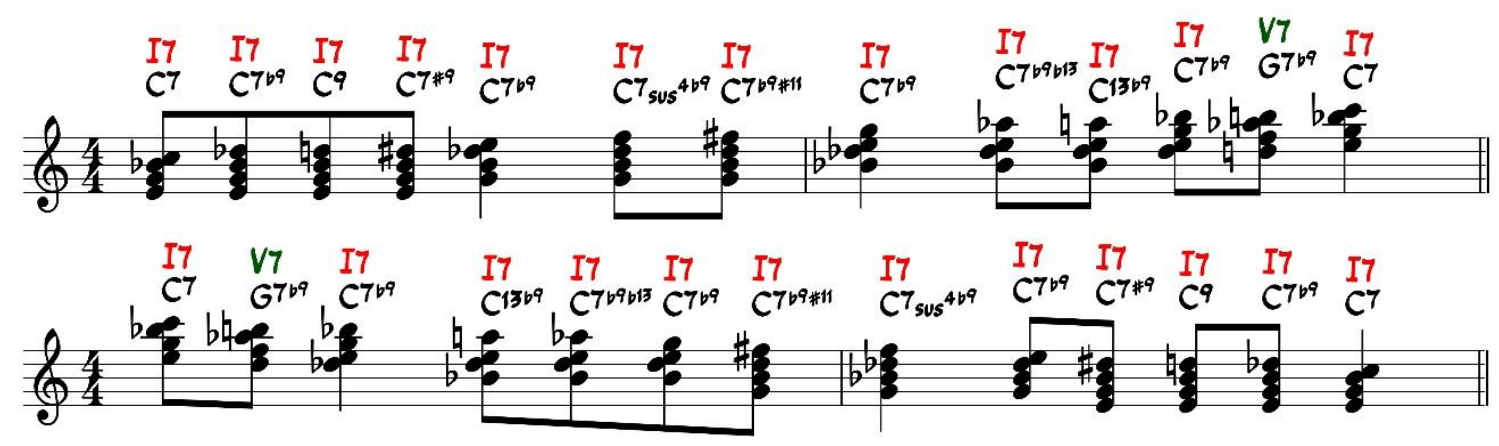

\subsection{Practical Applications}

We propose two solutions for the harmonization of the so-called Cataldo's Fundamental "Sentence" for Dominant Seventh Chords [10,14]: 


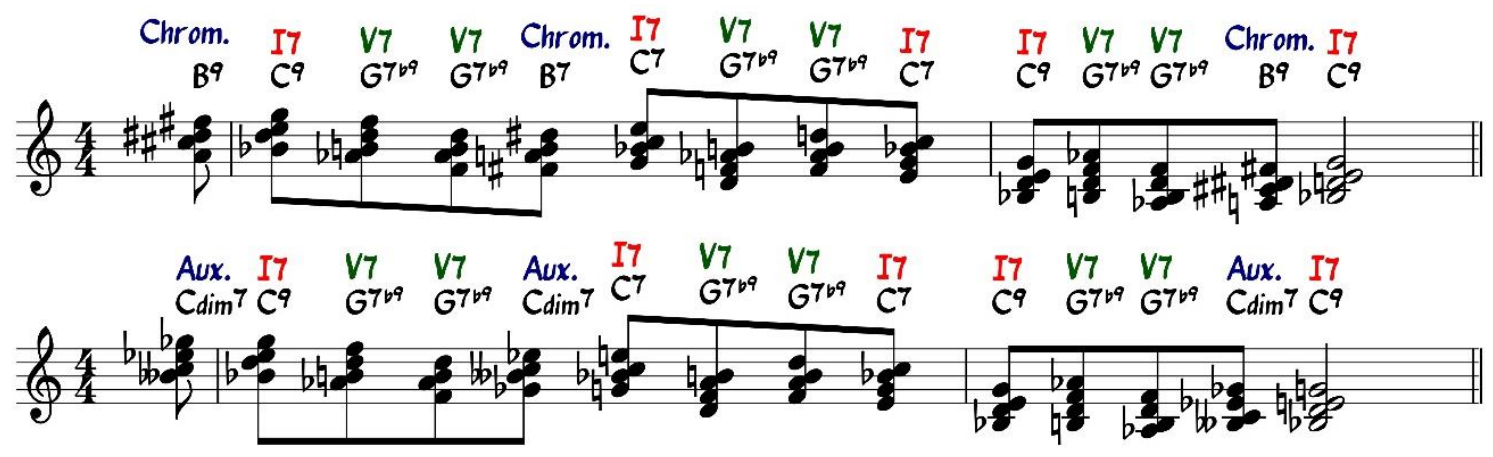

\section{Minor Sixth/Seventh Chords}

\subsection{Harmonic Flows}

The suggested harmonic flows are shown in the following diagram (Figure 3):

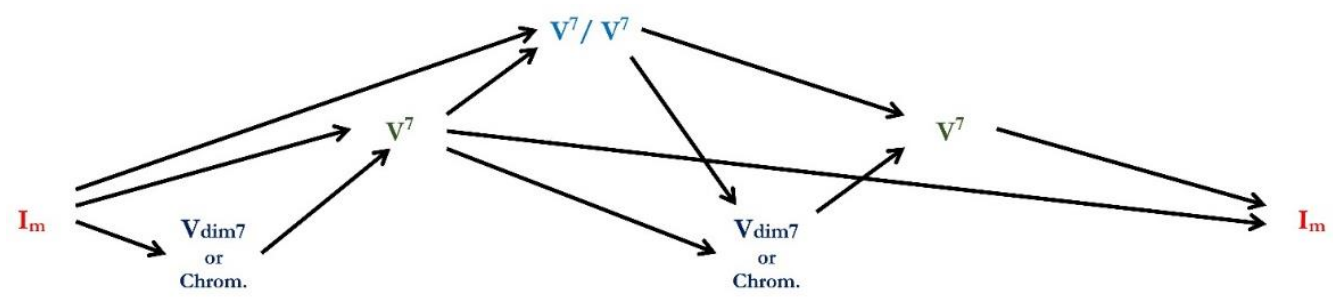

Fig.3 - Minor Sixth/Seventh Chords: Suggested Harmonic Flows

4.2. Voicings on Conventional Scales: Be-Bop Ipoionian (Melodic Minor) and Be-Bop Aeolian (Natural Minor)

In Staff (30) we have the conventional $4 \mathrm{WC}$ of the C Be-Bop Ipoionian Scale $[2,10]$ :

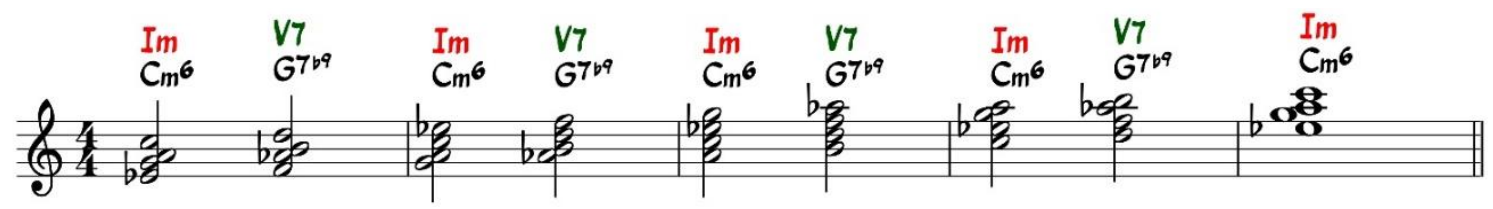

In Staff (31) we have the conventional 4WC of the C Be-Bop Aeolian Scale $[2,10]$ :

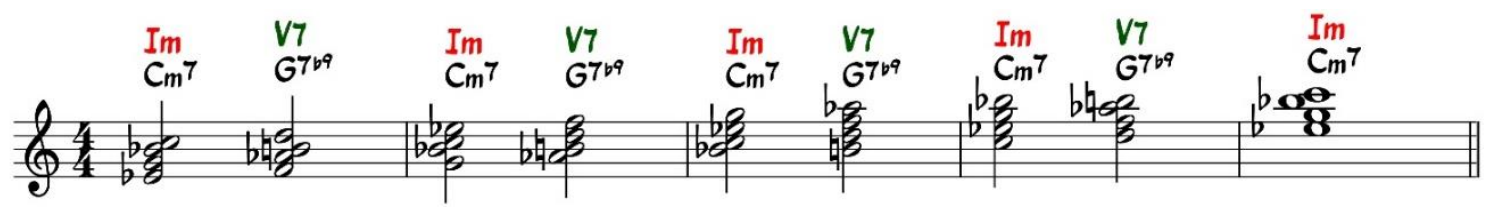

From Staff (30), by removing the cluster involving the lower part of the voicings built on the median (3rd), the underlying "hybrid" form is deduced [3]:

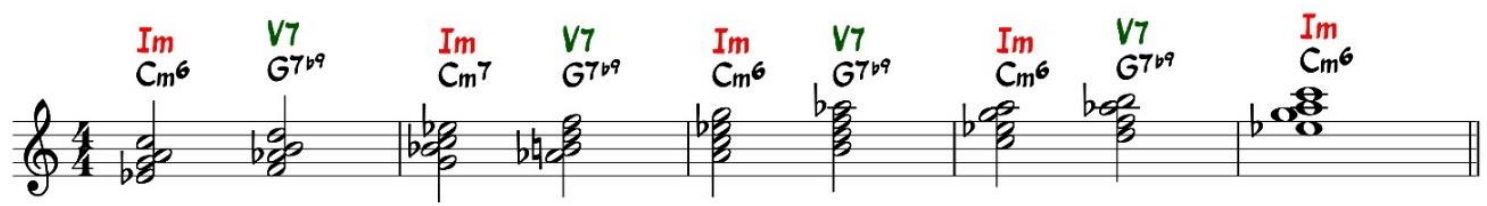


From Staff (31), by removing the cluster involving the lower part of the voicings built on the dominant (5th), the underlying "hybrid" form is obtained:

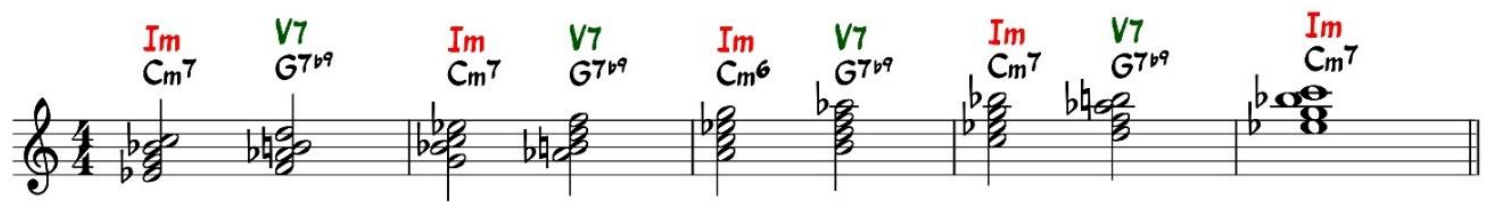

By solely modifying the voicing built on the dominant (labelled as Cm6), from Staves (32) and (33) the following alternative "hybrid" harmonizations are deduced:

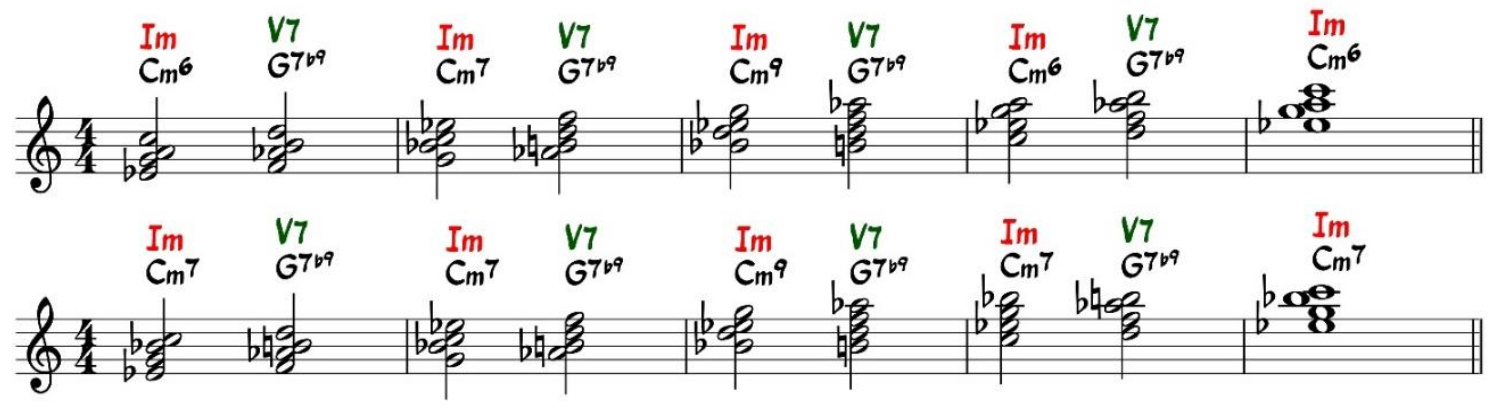

\subsection{Chromatic Scale}

From Staff (32), by resorting to Chromatic Parallelism, Diminished Auxiliary Chords and Tonicization, we obtain the underlying solution:

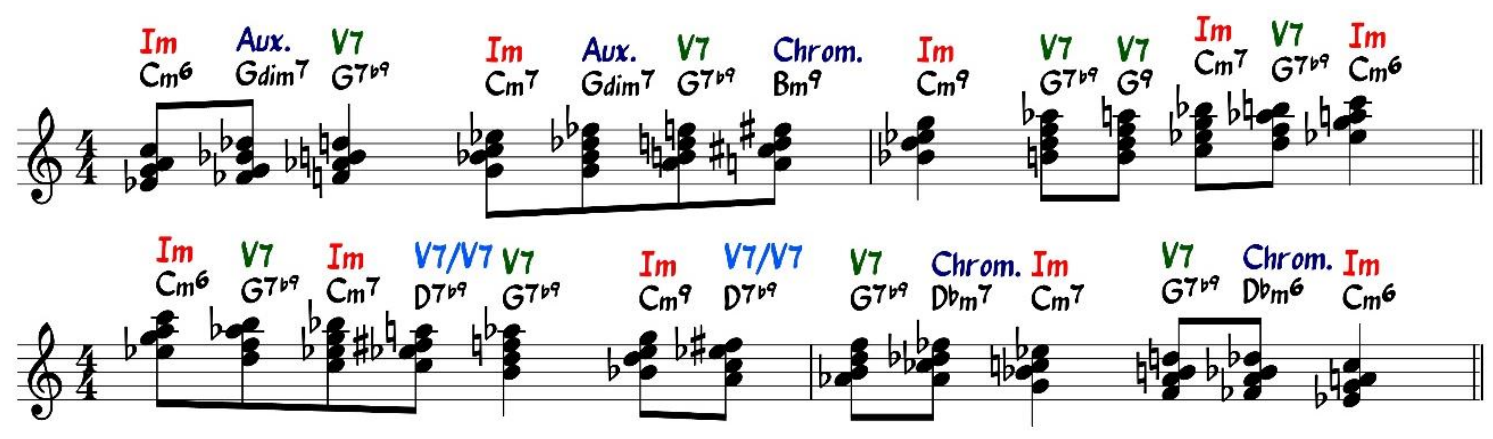

By starting, once again, from Staff (32), the following alternative solution is deduced:

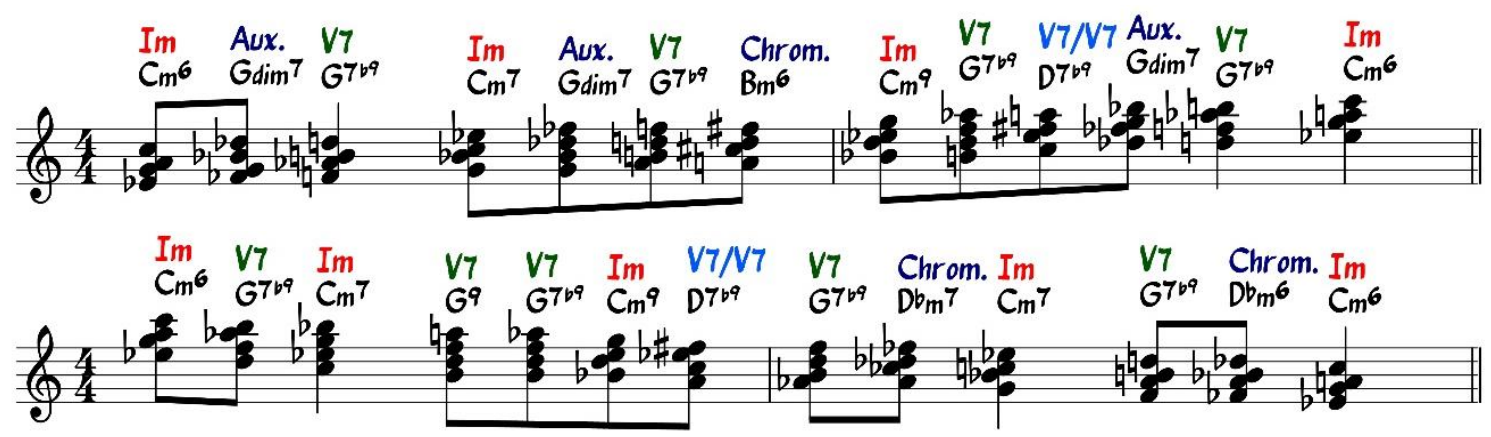

By starting from Staff (34), we obtain the following further harmonization: 


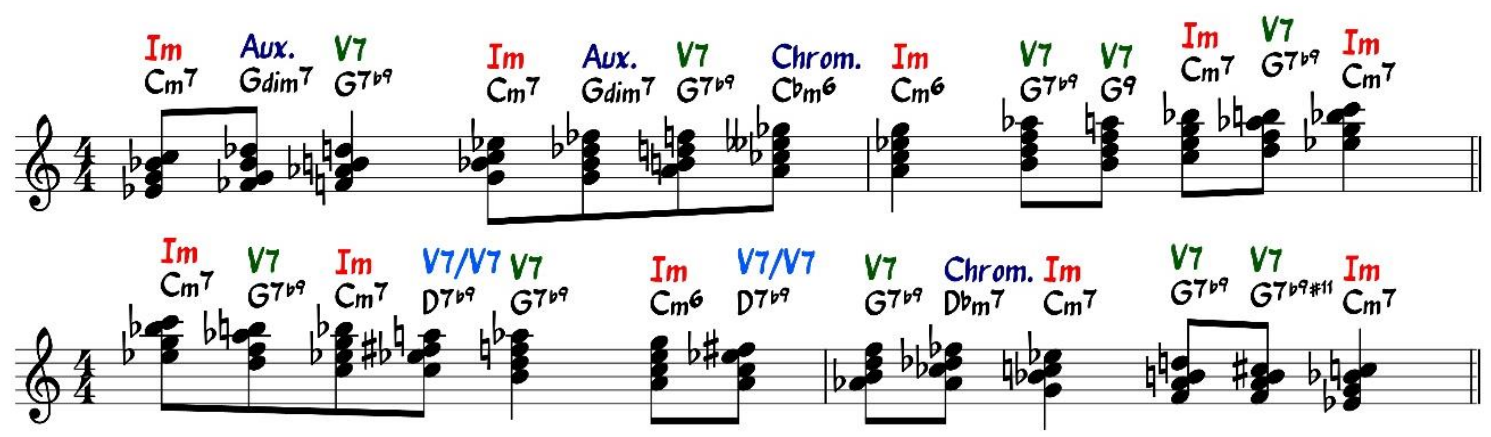

\subsection{Practical Applications}

We propose a single solution for the harmonization of the so-called Cataldo's Fundamental "Sentence" for Minor Sixth/Seventh Chords [10,15] (Cataldo, 2018a, 2017b):

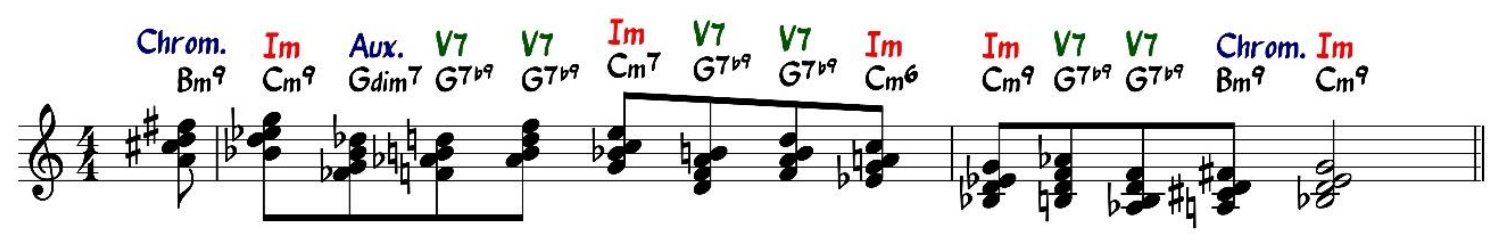

\section{Half Diminished Chords (in brief)}

For the sake of brevity, we herein limit ourselves to suggesting a single solution, which can be evidently regarded as Quasi-Static:

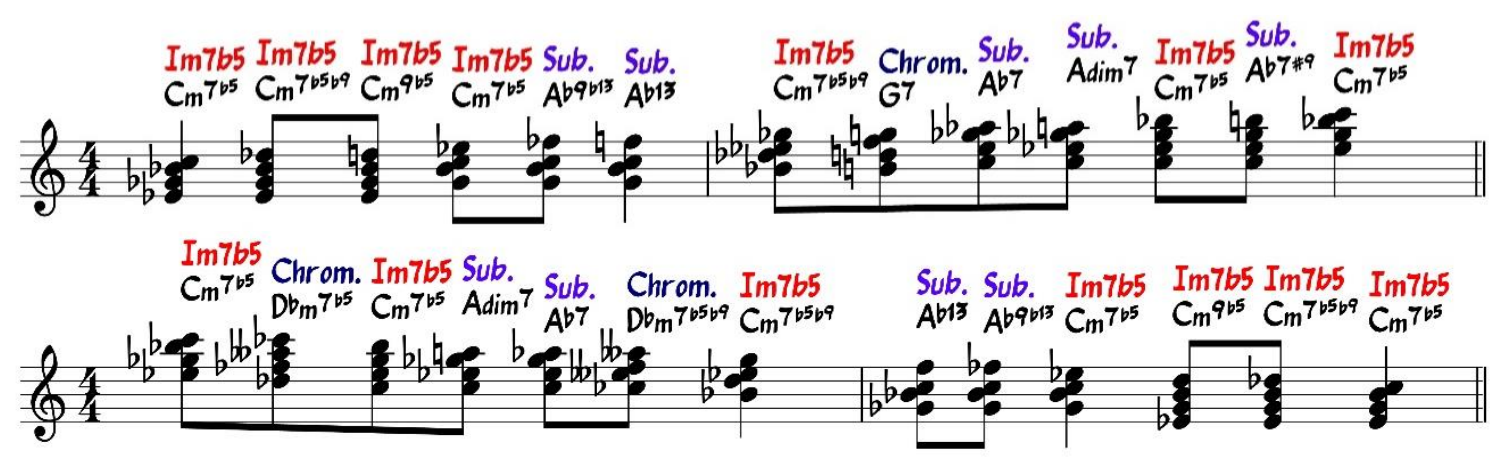

The voicings labelled as Sub. are obtained by simply resorting to Diatonic Substitutions $[7,12,13]$. Referring to the particular case in Staves (43) and (44), if the chord Cm7b5 is, as is usually the case, considered as being associated with the Locrian Mode, it can be replaced by $A b 7$ (Mixolydian). Similarly, if the chord $C m 7 b 5$ is considered as being associated to the Locrian \#6 Mode, it can be replaced by Adim7 (Ultralocrian) [4].

\section{Final Remarks and Conclusions}

Obviously, the solutions introduced herein are not exhaustive: therefore, any further harmonization complying with the suggestions listed in Paragraph 1 is fully admissible.

All the Staves herein proposed can be subjected to a dropping procedure $[3,8,9]$.

As an example, the DROP-2 version of Staff (11), extremely useful to jazz guitarists, yields the following: 


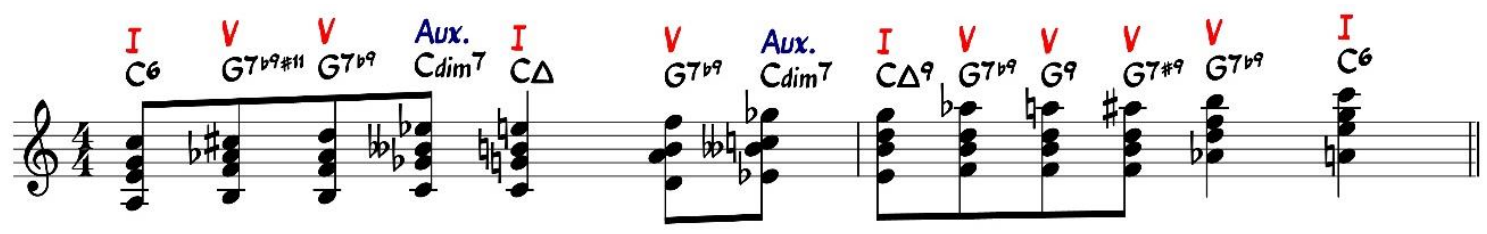

If the arrangement is intended for an ensemble, two consecutive notes, identical in pitch, should always be considered as being tied, at least as far as the Quasi-Static Solutions are concerned. In particular, the Quasi-Static Solutions, played exactly as they are written, are predominantly suitable for the jazz pianist interested in the so-called "lockedhands" technique.

Solutions suitable for Diminished Chords can be deduced by slightly modifying the harmonization proposed in Paragraph 5. Solutions suitable for Minor Major Seventh Chords can be deduced by slightly modifying the harmonizations proposed in Paragraph 4. Solutions suitable for Augmented Major Seventh Chords can be deduced by slightly modifying the harmonizations proposed in Paragraph 2.

The understanding of the method introduced herein, based on the simple rules listed in Paragraph 1, allows one to effectively deal with 4WC Harmonization of non-diatonic melodic lines built on the most common jazz progressions, such as IIm7 | V7 | Imaj7 (or $\operatorname{Im} 7)$ and $\operatorname{IIm} 7 b 5|\mathrm{~V} 7| \operatorname{Im} 7$ (or Imaj7).

Acknowledgments: The author would like to thank Rawl Gelinas, engineer and jazz musician, for the English proofreading of this paper.

\section{References}

1. Levine, M. The Jazz Theory Book (Italian Edition by F. Jegher). Milan, IT: Curci Jazz, 2009.

2. Baker, D. How to Play Bebop (Volume 1). Los Angeles, CA: Alfred Publishing Co. Inc., 1988

3. Cataldo, C. Jazz Arranging for Beginners - Part 2 [Tecniche di Arrangiamento Jazz per Principianti - Seconda Parte]. Journal of Science, Humanities and Arts (JOSHA), 7(2), 2020. https://dx.doi.org/10.17160/josha.7.2.644

4. Cataldo, C. The Evolution of Harmonic Progression Analysis: Ultimate CAT. Journal of Science, Humanities and Arts (JOSHA), 5(7), 2018. https://dx.doi.org/10.17160/josha.5.7.469

5. Cataldo, C. Extreme Chord Substitutions: a Qualitative Introduction to CAT (Cataldo Advanced Transformations). Journal of Science, Humanities and Arts (JOSHA), 5(4), 2018. https://dx.doi.org/10.17160/josha.5.4.424

6. Nettles, B. Harmony 3 - Spring 2007 Edition. Berklee College of Music, 2007

7. Cho, G. J. Theories and Practice of Harmonic Analysis. Lewiston, NY: E. Mellen Press, 1992.

8. Cataldo, C. Jazz Arranging for Beginners - Part 1 [Tecniche di Arrangiamento Jazz per Principianti - Prima Parte]. Journal of Science, Humanities and Arts (JOSHA), 7(2), 2020. https://dx.doi.org/10.17160/josha.7.2.642

9. Dobbins, B. Jazz Arranging and Composing - L'Approccio Lineare (Italian Ed. by R. Spadoni). Italy: Volontè \& Co., 2010

10. Cataldo, C. Breve Introduzione all'Improvvisazione Be-Bop [Short Introduction to Be-Bop Improvising]. Journal of Science, Humanities and Arts (JOSHA), 5(6), 2018. https://dx.doi.org/10.17160/josha.5.6.448

11. Cataldo, C. The Art of Improvising: the Be-Bop Language and the Major Seventh Chords. Art and Design Review, 5, 222-229, 2017. https://doi.org/10.4236/adr.2017.54018

12. Cataldo, C. Music Algebra: Harmonic Progressions Analysis and CAT (Cataldo Advanced Transformations). International Journal of Advanced Engineering Research and Science, 5(5), 224-227, 2018. https://dx.doi.org/10.22161/ijaers.5.5.29

13. Cataldo, C. Towards a Music Algebra: Fundamental Harmonic Substitutions in Jazz. International Journal of Advanced Engineering Research and Science, 5(1), 178-181, 2018. https://dx.doi.org/10.22161/ijaers.5.1.9

14. Cataldo, C. The Art of Improvising: the Be-Bop Language and the Dominant Seventh Chords. Art and Design Review, 5, 181-188, 2017. http://doi.org/10.4236/adr.2017.53014

15. Cataldo, C. The Art of Improvising: the Be-Bop Language and the Minor Seventh Chords. Art and Design Review, 5, 213-221, 2017. https://doi.org/10.4236/adr.2017.54017 